\title{
Phase and density dependence of the delayed annihilation of metastable antiprotonic helium atoms in gas, liquid, and solid helium
}

\author{
E. Widmann, ${ }^{*}$ I. Sugai, and T. Yamazaki \\ Institute for Nuclear Study, University of Tokyo, 3-2-1 Midori-cho, Tanashi, Tokyo 188, Japan \\ R. S. Hayano, M. Iwasaki, S.N. Nakamura, H. Tamura, T.M. Ito, and A. Kawachi \\ Department of Physics, University of Tokyo, 7-3-1 Hongo, Bunkyo-ku, Tokyo 113, Japan \\ N. Nishida and W. Higemoto \\ Tokyo Institute of Technology, O-Okayama, Meguro-ku, Tokyo 152, Japan \\ Y. Ito \\ Research Center for Nuclear Science and Technology, University of Tokyo, Tokai, Ibaraki 319-11, Japan \\ N. Morita \\ Institute for Molecular Science, Myodaiji, Okazaki 444, Japan \\ F. J. Hartmann, H. Daniel, T. von Egidy, W. Schmid, and J. Hoffmann \\ Physik-Department, Technische Universität München, D-85747 Garching, Germany \\ J. Eades \\ CERN, CH-1211 Geneva 23, Switzerland \\ (Received 6 December 1994)
}

\begin{abstract}
Systematic studies of the phase and density dependence of the delayed annihilation time spectra of antiprotons (DATS) in gaseous, liquid, and solid helium have been performed using the low energy antiproton ring at CERN. The results show a small but significant dependence of DATS on the phase and density of the helium medium. The average lifetime of antiprotons $\left(T_{a v}\right)$ in gas at 10-30 $\mathrm{K}$ was found to be $4.03 \pm 0.02 \mu \mathrm{s}$. In liquid helium $T_{a v}$ decreases with density from $3.0 \mu \mathrm{s}$ at 1 bar to $2.5 \mu \mathrm{s}$ at $60 \mathrm{bar}$, while in solid helium its value is $2.14 \pm 0.03 \mu \mathrm{s}, 20 \%$ shorter than that in liquid helium of the same density. There is no change of DATS between normal 1-bar liquid and superfluid helium. An isotope effect between ${ }^{4} \mathrm{He}$ and ${ }^{3} \mathrm{He}$ similar to the one previously observed in the gas phase has also been found for liquid helium. All the data can be fitted fairly well with a simple three-level chain decay model which describes the general structure of DATS, characterized, by downward curving behavior on a logarithmic scale at later times and by the presence of a short-lived component. No delayed annihilation was observed in lithium.
\end{abstract}

PACS number(s): 36.10.-k

\section{INTRODUCTION}

Metastable antiprotonic helium atoms were first discovered in an experiment at KEK [1] where about $3 \%$ of antiprotons stopped in liquid helium were found to survive for an average lifetime of about $3 \mu \mathrm{s}$. Similar but much shorter-lived metastable states had been found for $\mathrm{K}^{-}[2]$ and $\pi^{-}[3]$ in liquid helium. No evidence for metastable states in liquid nitrogen or argon has been found.

The observed longevity is in contradiction to the common belief that negatively charged hadrons stopped in matter are immediately (i.e., within $10^{-12} \mathrm{~s}$ ) captured

${ }^{*}$ Present address: CERN, CH-1211 Geneva 23, Switzerland. by the nucleus. The metastability can be attributed to the formation of long-lived states in exotic helium atoms $\bar{p} e^{-} \mathrm{He}^{2+}\left(=\bar{p} \mathrm{He}^{+}\right)$. The antiproton $(\bar{p})$ is expected to be bound in one of the nearly "circular" states, i.e., states with large principal quantum number $n \approx n_{0}=$ $\sqrt{M^{*} / m_{e}}\left(M^{*}\right.$ being the reduced mass and $m_{e}$ the mass of the electron) and large orbital angular momentum $l \sim n_{0}-1$. In this region, since the level spacings $(\approx$ $2 \mathrm{eV})$ are much smaller than the ionization energy $(\approx$ $25 \mathrm{eV}$ for helium), fast Auger transitions with a small change of $l$ are energetically impossible, while the radiative transitions proceed very slowly. Also, the presence of the electron and the resulting neutrality of the exotic atom removes the degeneracy of different- $l$ states for the same $n$ and helps suppress deexcitation via Stark mixing.

This model was first suggested by Condo [4] to explain the anomalous free-decay fractions of $\pi^{-}$and $\mathrm{K}^{-}$ 
observed in liquid helium bubble chambers and later extended by Russell [5], who also discussed the case of antiprotons (for historical accounts, see [6]). The energy levels and radiative rates have been calculated recently on the basis of this $\bar{p} \mathrm{He}^{+}$atomic model using a configuration mixing approach [7] as well as a molecular approach [8-10]. The results of the calculations are mutually consistent and give typical single-level lifetimes in the 1-2 $\mu \mathrm{s}$ range. Obviously, however, these are not the total decay rates of individual states because of the competing Auger processes nor are they directly connected to the survival time. It is shown in a recent calculation of Ohtsuki $[11,12]$ that only those states which can proceed to lower-lying ionized states $\left(\bar{p} \mathrm{He}^{2+}\right)$ via $\Delta l \leq 3$ Auger emission are short lived (lifetime shorter than $10 \mathrm{~ns}$ ), while the others are radiation-dominated long-lived states. Thus the $(n, l)$ energy level diagram is divided into a metastable zone and a short-lived zone, as shown in $[7,13]$. Recent laser resonance experiments $[14,15]$ have confirmed these calculations of the overall structure of metastable states of this $\bar{p} \mathrm{He}^{+}$atom and its Auger-dominated short-lived states.

Observed delayed annihilation time spectra of antiprotons (DATS) are in general the results of cumulative effects of series of metastable cascades and thus involve not only the single-level decay rates but also their initial populations and subsequent quenching processes by atoms of the surrounding medium. These features cannot be separately understood just by examining the shape of the DATS. On the other hand, the calculations are only valid for the structure of an isolated atom and do not take into account either the initial formation process or interactions with the atoms in the surrounding medium. If there is a cascade of several transitions before the $\bar{p}$ reaches a state from which it is captured by the nucleus and if furthermore there is no collisional quenching effect, the single-level lifetimes of $1-2 \mu$ s should result in a much longer trapping time than the observed $3 \mu \mathrm{s}$.

This discrepancy prompted us to measure the DATS of $\bar{p}$ in gaseous helium; the collision rate with other helium atoms is reduced, so that we might expected a significant increase of the trapping time in gaseous helium. On the contrary, the first experiments performed at the Low Energy Antiproton Ring (LEAR) at CERN $[13,16]$ showed only slight changes in the trapping time between liquid and room-temperature helium gas.

In the present paper we report on systematic studies of the phase and density dependence of DATS using the antiproton beam from LEAR, extending our measurements to low temperature ${ }^{4} \mathrm{He}$ gas, high pressure liquid ${ }^{4} \mathrm{He}$ and ${ }^{3} \mathrm{He}$, and solid ${ }^{4} \mathrm{He}$. Section II describes the experimental setup including the various cryogenic targets, Sec. III describes briefly the data analysis procedure, and Sec. IV gives the experimental results, which are discussed in Sec. V. In Sec. VI we give the conclusions we draw from the experiments.

\section{EXPERIMENTAL SETUP}

In this paper we describe measurements of DATS in helium media under the following conditions: (i) low temperature ${ }^{4} \mathrm{He}$ gas at $T=10$ to $40 \mathrm{~K}$ and $p$ $=1 \mathrm{bar}$, (ii) liquid ${ }^{4} \mathrm{He}$ at $T=1.5$ to $3.5 \mathrm{~K}$ and $p=1$ to $60 \mathrm{bar}$, (iii) liquid ${ }^{3} \mathrm{He}$ at $T=2.5 \mathrm{~K}$ and $p=1$ to 30 bar, and (iv) solid ${ }^{4} \mathrm{He}$ at $T=1.5 \mathrm{~K}$ and $p=50.5$ bar.

Our method of measuring DATS in gaseous helium at LEAR has already been described elsewhere in great detail [16]. The experiments reported here were performed at the $\mathrm{C} 1$ beam line of LEAR, using a $\bar{p}$ beam of momentum $p=200 \mathrm{MeV} / c$ (kinetic energy $T=21.1 \mathrm{MeV}$ ). This beam momentum made it easier to perform experiments on solid and high pressure liquid helium than was the case with our previous $100 \mathrm{MeV} / c$ beam because we could use thicker beam counters and windows. The target vessels were big enough to accommodate the concomitant disadvantages of longer range straggling in the low density gas targets and larger angular spread of the antiprotons in the degraders and windows.

The setup as shown in Fig. 1 is very similar to the one described in [16], except that the scintillators were placed vertically to allow the installation of cryostats for the low temperature measurements. The antiprotons left the beam line through a 100- $\mu \mathrm{m}$-thick Be window and entered our target chamber after passing through a $1-\mathrm{mm}$ thick plastic scintillation counter $(B)$ of $10 \mathrm{~mm}$ diameter $(18 \mathrm{~cm}$ downstream of the $\mathrm{Be}$ window). The $B$ counter was read out by two photomultiplier tubes operated in coincidence. An additional ring-shaped counter $(A)$, which served as a veto counter as well as a monitor of the incoming beam halo, was placed about $1 \mathrm{~cm}$ upstream of $B$. The targets and the detectors for charged annihilation products (mainly pions) were placed just downstream of the beam counters. The outgoing $\pi^{ \pm}$were detected by the same four sets of plastic scintillator telescopes used in previous experiments [16]. Each consisted of one inner $\left(T 1_{i}, i=A, B, C, D\right.$ in Fig. 1$)$ and three outer arrays $\left(T 2_{i j}, j=a, b, c\right)$ covering a solid angle of $0.8 \pi$ and was divided into 12 elements to reduce the probability of two or more pions hitting the same telescope counter. This was necessary to reduce the background which is caused by $\pi^{+} \rightarrow \mu^{+} \rightarrow e^{+}$decay from pions stopped in the vicinity of the target chamber. These give a background with a lifetime of $2.2 \mu \mathrm{s}$, which lies within the time scale of interest. In order to reduce this background, it was found to be very effective to tag multipion hits in the telescope counters [16].

\section{A. Low temperature gas target}

To provide gas samples at temperatures between $10 \mathrm{~K}$ and $40 \mathrm{~K}$, we used a helium-flow cryostat specially designed for this purpose (Fig. 2). The horizontal cylindrical target chamber had a diameter of $6 \mathrm{~cm}$ and a length of $18 \mathrm{~cm}$. It was surrounded by an outer cylindrical chamber through which cold helium gas flowed, this being produced by vaporizing some of the liquid supplied from the liquid helium reservoir through a needle valve. The temperature of the cooling gas was controlled by a heater located close to the vaporizer at the entrance of the outer chamber. The $\bar{p}$ entered the target chamber through two 


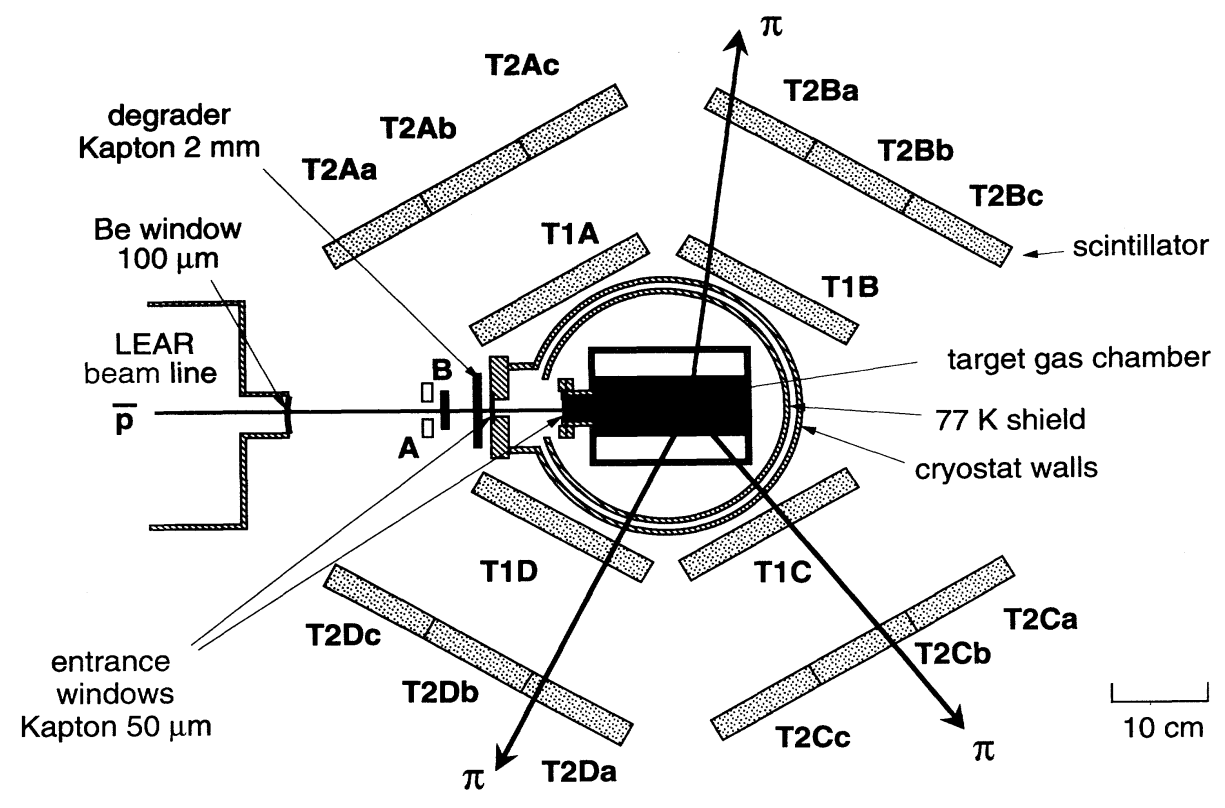

FIG. 1. Setup to measure delayed annihilation time spectra of antiprotons in helium (top view). The $B$ and $A$ counters have been drawn on an enlarged scale. In the center the cryostat for low temperature gas measurements is shown.

Kapton windows of $50 \mu \mathrm{m}$ thickness and $2 \mathrm{~cm}$ diameter. One was located at the vacuum layer and the other one at the target chamber. An additional Kapton degrader of $2 \mathrm{~mm}$ thickness was necessary. This was placed outside the vacuum, which caused a fraction of the $\bar{p}$ to stop outside the target chamber window due to multiple scattering. The typical range distribution of stopped $\bar{p}$ was about $3 \mathrm{~cm}$ full width at half maximum (FWHM) in 1 bar and $10 \mathrm{~K}$ gas and the transverse size was about $2 \mathrm{~cm}$ FWHM.

The inner target chamber was filled with research grade (impurity content $<1 \mathrm{ppm}){ }^{4} \mathrm{He}$ gas from a gas bottle through standard quick coupling vacuum bellows with rubber seals. No effort was made to bake the gas handling system since we expected all possible impurities to be frozen out at temperatures well below liquid nitrogen temperature. The cryostat construction limited the pressure of the target cell to 1 bar.

\section{B. Cryostat for liquid and solid helium}

Another cryostat was made for producing liquid and solid helium. In this case the helium target size was 12 $\mathrm{mm}$ in diameter and $11 \mathrm{~mm}$ in length; the total amount of target material was about 0.1 mole. The target chamber was made of copper with a $\mathrm{Cu}-\mathrm{Be}$ alloy beam window of $12 \mathrm{~mm}$ diameter and $0.49 \mathrm{~mm}$ thickness. The window was sealed by an indium wire and was able to withstand more than 90 bars of pressure at liquid helium temperature. The target chamber was surrounded by two copper walls at $4.2 \mathrm{~K}$ and $77 \mathrm{~K}$ acting as radiation shields (see Fig. 3). Antiprotons were introduced into the liquid or solid helium through four thin windows: a $120-\mu \mathrm{m}$-thick Kapton foil window at room temperature, a 50- $\mu$ m-thick aluminized Mylar foil window at $77 \mathrm{~K}$, a 20- $\mu \mathrm{m}$ copper foil at $4.2 \mathrm{~K}$, and the $0.49-\mathrm{mm}$-thick $\mathrm{Cu}$-Be window of the

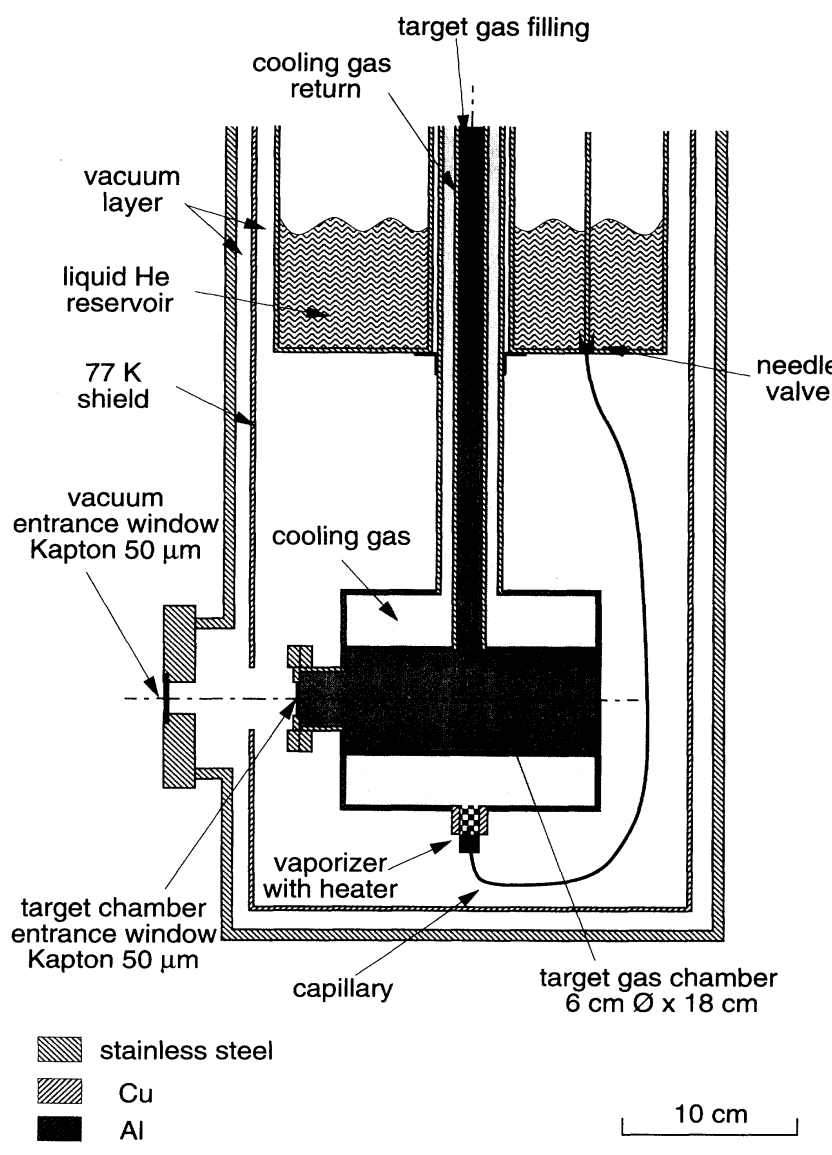

FIG. 2. Cryostat for low temperature gas measurements (side view). In the upper part the liquid helium reservoir with a needle valve to control the flow of liquid helium through the capillary to the vaporizer is shown. In the lower part the target gas chamber and the surrounding cooling gas chamber are seen. The antiproton beam came from the left-hand side. 


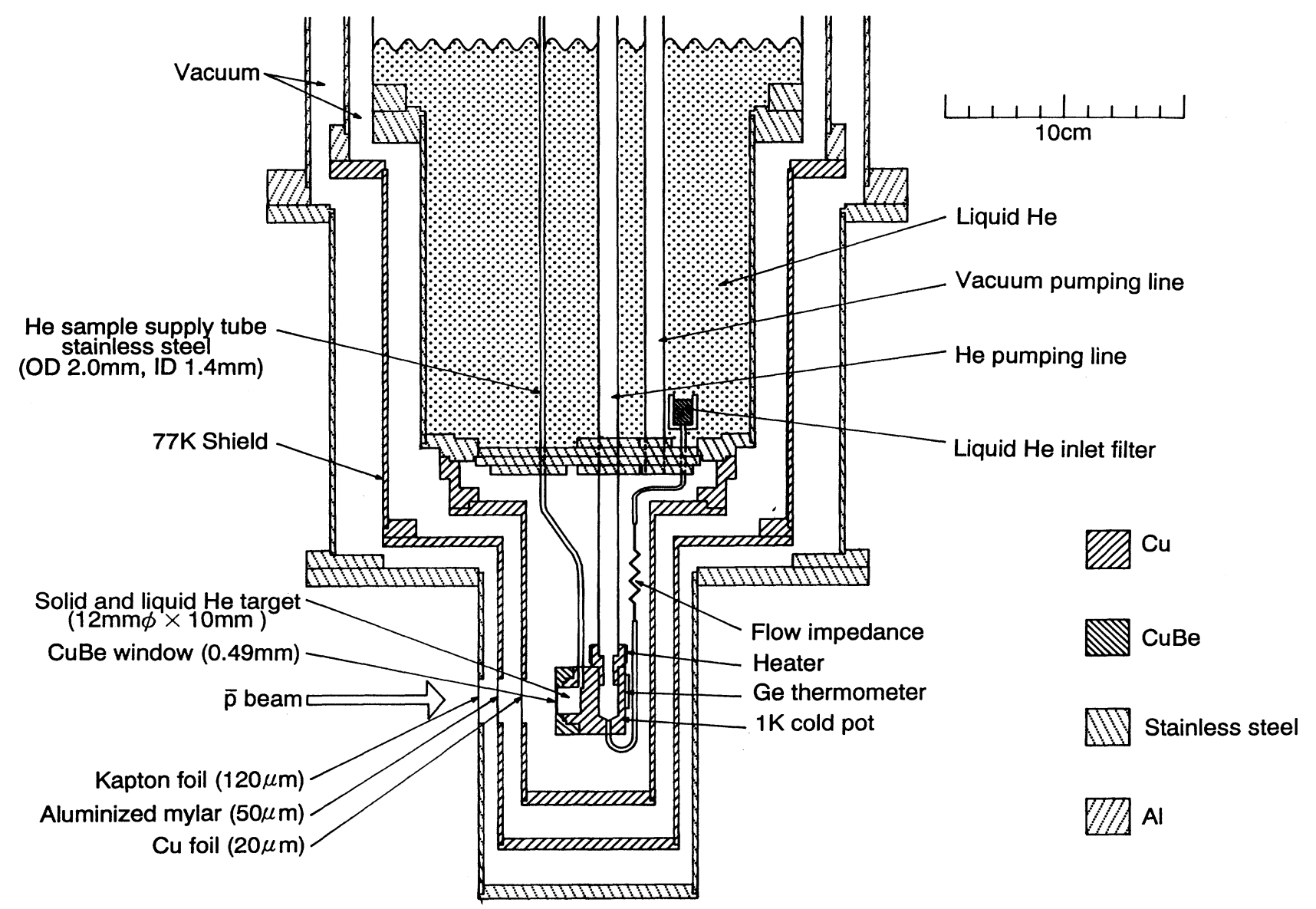

FIG. 3. Detailed view of the cryostat for solid and high pressure liquid helium. For details see the text.

target chamber. As with the low temperature gas cryostat, a small fraction of the antiproton beam fell outside the entrance window and therefore stopped in the walls of the target vessel.

Helium gas was introduced into the target chamber through a stainless steel tube (outside diameter $2.0 \mathrm{~mm}$ and inside diameter $1.4 \mathrm{~mm}$ ) and was liquefied at the target chamber or solidified there by pressurizing it in a conventional way by using a dip-stick which contained about $6 \mathrm{~g}$ of activated charcoal and was able to absorb about 0.16 mole of helium when the dip-stick was immersed into liquid helium. The gas handling system is schematically shown in Fig. 4. Temperatures between $1.5 \mathrm{~K}$ and $4.2 \mathrm{~K}$ were achieved by pumping and introducing liquid helium into the $1 \mathrm{~K}$ "cold pot" through a flow impedance (Fig. 3).

\section{DATA REDUCTION AND ANALYSIS}

The electronics and data taking procedure have been described in great detail in a previous publication [16]. We measured the time difference between an incoming antiproton ( $B$ counter hit) and an outgoing annihilation product (a hit in at least one of the 12 telescope elements). We took separate time spectra for each counter, so that in the analysis we could select multipion hits to discriminate the background of the $\pi^{+} \rightarrow \mu^{+} \rightarrow e^{+}$decays mentioned above. In a previous analysis [16] we selected events where at least three pions were detected within a time window of $3.3 \mathrm{~ns}$ [referred to as (multiplicity) $\geq 3$, or M3 events] to obtain the final time spectra. This method, however, leads to a significant decrease of statistics. In the present analysis, we chose a different approach, taking data with an empty target for each experimental setup (low temperature gas, or liquid or solid helium) and subtracting the M2 [(multiplicity) $\geq 2]$ spectra (normalized to the same total counts) from the spectra with helium in the target to obtain time spectra without the $\pi^{+} \rightarrow \mu^{+} \rightarrow e^{+}$decay background.

Before doing this we had to correct the M2 time spectra [cf. Fig. 5(b)] for a time-independent background arising from the inefficiency of the beam counter, which failed to detect a small fraction of "second" $\bar{p}$ following a given $\bar{p}$ within our $50-\mu$ s observation time "window" (see [16]). The level of this background depends on the threshold of the discriminator on the $B$ counter, the beam alignment, and the $\bar{p}$ intensity. It was defined as the average count per bin in the range $25 \mu \mathrm{s}<t<45 \mu$ s and subtracted for each run separately. Figure 5(c) shows the spectrum of Fig. 5(b) after subtraction of this constant background. Figure $5(\mathrm{~d})$ presents the remaining spec- 


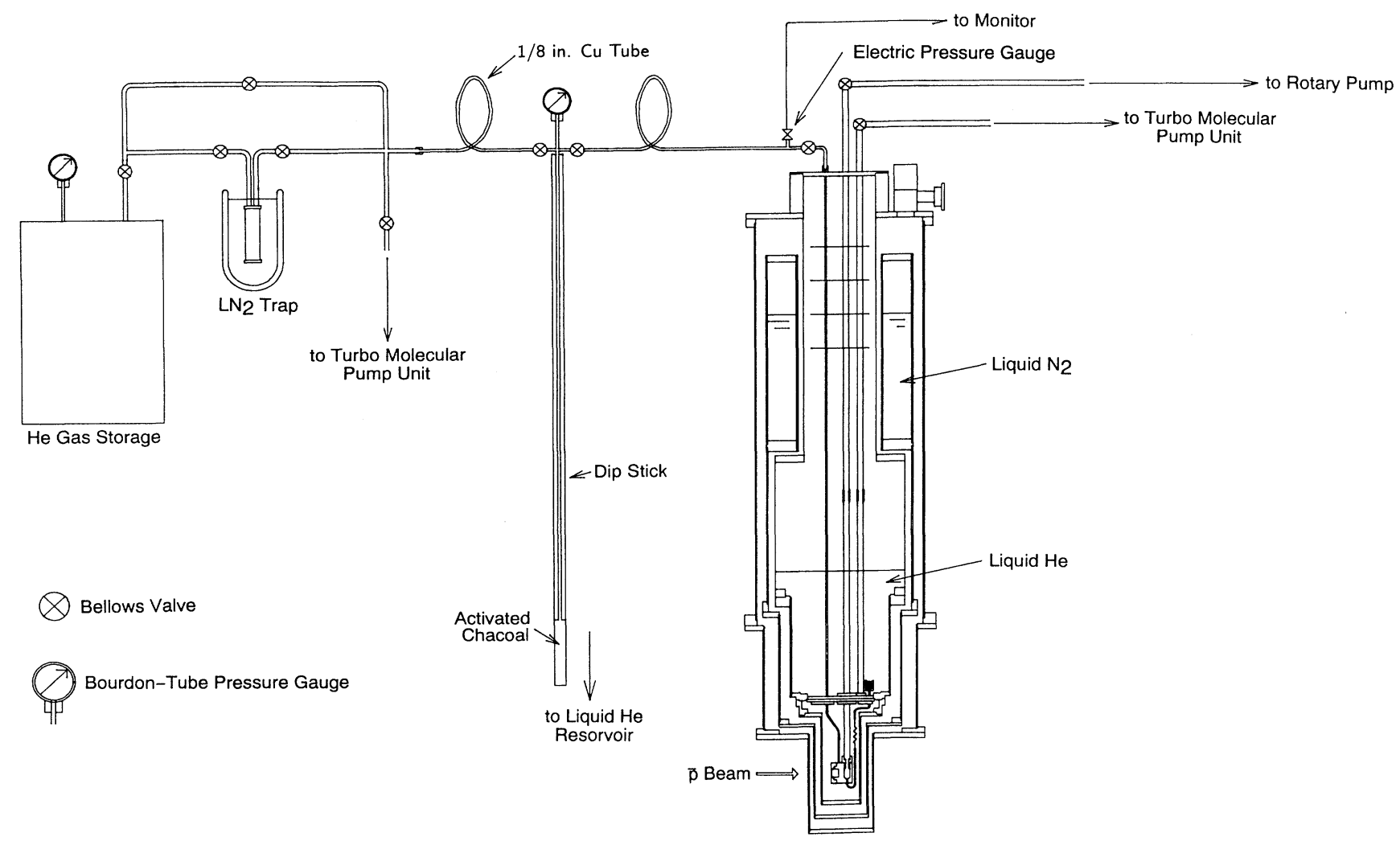

FIG. 4. Complete system for the production of solid $\mathrm{He}^{4}$.

trum after subtraction of the histogram of the empty run [Fig. 5(a)]. Due to the subtraction, Fig. 5(d) no longer shows a prompt peak.

All spectra presented in this paper have been treated in this manner. In addition they have been normalized to one to allow for a direct comparison of spectra taken under different conditions and with different statistics.

\section{EXPERIMENTAL RESULTS}

Tables I and II give an overview of the experimental conditions and results for low temperature ${ }^{4} \mathrm{He}$ gas, liquid and solid ${ }^{4} \mathrm{He}$, and liquid ${ }^{3} \mathrm{He}$. The tables give the temperature and pressure of the medium as well as the molar volume $V_{m o l}$ and the number density $\rho$ at each condition. The number density was chosen as a parameter that represents the effect of collisions. For low temperature gas measurements, experimental data for the second virial coefficient [17] were used to calculate $V_{m o l}$, although above $T=10 \mathrm{~K}$ the difference from using the ideal-gas equation is smaller than the uncertainties in measuring and stabilizing the pressure and temperature inside the cryostat. For liquid ${ }^{4} \mathrm{He}$ and ${ }^{3} \mathrm{He}$ the values were taken from experimental data [18]. Typical spectra (obtained in the way previously described) corresponding to different conditions are shown in Figs. 6 and 7 . We chose a binning of $100 \mathrm{~ns}$ for these displays, though the intrinsic time resolution of our time spectra is $3 \mathrm{~ns}$.
The average lifetime $T_{a v}$ used to characterize the spectra $N(t)$ was calculated as follows:

$$
T_{a v}\left(t_{0}\right)=\frac{\int_{t_{0}}^{t_{\max }} t N(t) \mathrm{d} t}{\int_{t_{0}}^{t_{\max }} N(t) \mathrm{d} t}-t_{0}
$$

where $t_{\max }$ was fixed at $25 \mu \mathrm{s}$. Both $T_{a v}(150 \mathrm{~ns})$ and $T_{a v}$ $(1 \mu \mathrm{s})$ are presented in Tables I and II. Hereafter, $T_{a v}$ without specified $t_{0}$ is the one for $t_{0}=1 \mu \mathrm{s}$. The tables do not give any values for the trapping fraction since the high beam momentum and the small diameters of the windows required by the high pressure of the targets caused a significant fraction of the particles to stop in the surrounding material.

Figure 6 shows the DATS in helium gas obtained below $40 \mathrm{~K}$ plotted logarithmically. They show the longest lifetime observed so far $\left(T_{a v} \approx 4 \mu \mathrm{s}\right)$. The bending-down behavior at times $t>10 \mu \mathrm{s}$, which was already found in previous measurements with room temperature helium gas [16], is seen very clearly. This behavior cannot be given by a simple sum of exponentials and suggests a sequential decay of metastable states.

The spectra for low temperature gas differ in one feature from the spectra under all other conditions: almost no fast-decaying component is visible below $30 \mathrm{~K}$. At 40 $\mathrm{K}$, however, there was some indication of a very fast component, while at temperatures of $50 \mathrm{~K}$ and above the time spectrum changed drastically, showing a mean lifetime of 


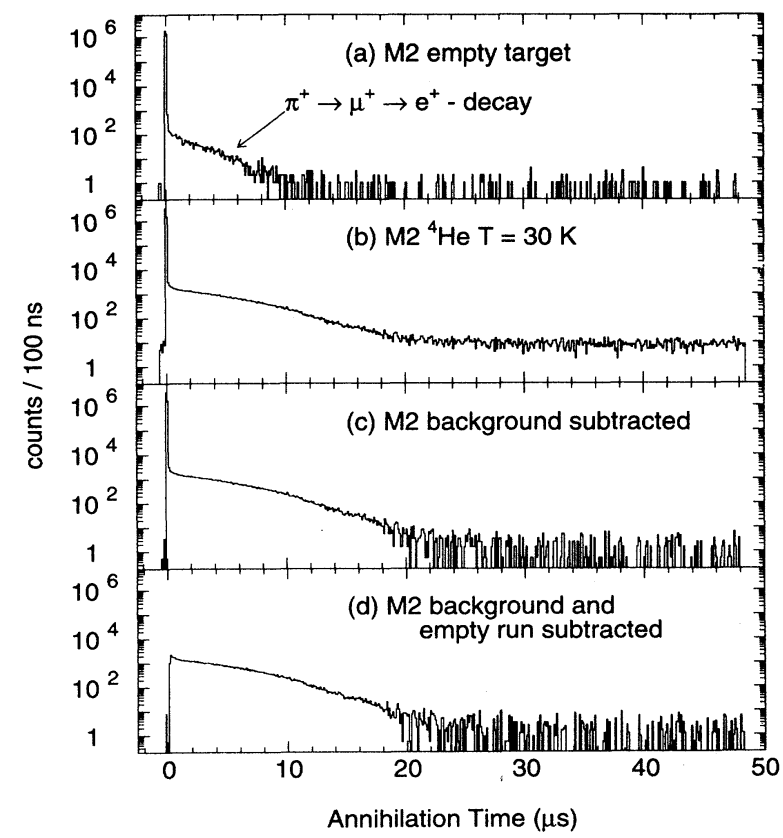

FIG. 5. Examples showing different steps in the data analysis procedure for $30 \mathrm{~K}$ He gas. (a) M2 DATS spectrum of the empty target. Antiprotons annihilate in the downstream wall of the target chamber, where they produce only prompt annihilations. The visible component with about $2.2 \mu \mathrm{s}$ lifetime is due to muon decays originating from positive pions stopped in the vicinity of the target. (b) M2 spectrum of $30 \mathrm{~K} \mathrm{He}$ gas showing a time constant background at $t>24 \mu \mathrm{s}$. (c) The same histogram after subtraction of the time constant background. (d) Final histogram after subtraction of the empty run (a) from histogram (c).

only a few hundred nanoseconds. This is most probably due to some impurity which started to evaporate around $50 \mathrm{~K}$ (both oxygen and nitrogen have considerable vapor pressures in this temperature range [19] and hydrogen liquefies only at $20.4 \mathrm{~K}$ ). The presence of a small quantity of impurities inside the target chamber cannot be excluded since we were not able to bake it in situ. We therefore use only the data below $30 \mathrm{~K}$ for the following discussions.

Figure 7 shows typical DATS of liquid and solid ${ }^{4} \mathrm{He}$ at different pressures. The spectra have a short-lived component and at higher pressure an almost straight exponential behavior at later times. Since the temperature is always below $5 \mathrm{~K}$, no impurities can be present and the presence of the short-lived component as well as the shorter $T_{a v}$ must be a pressure-related effect.

We have also measured DATS in solid $\mathrm{Li}$ metal, $\mathrm{LiH}$, and LiF. Typical data are shown in Fig. 8. No delayed component was found for any of the $\mathrm{Li}$ targets, the upper limit of the delayed fraction being $0.015 \%$, which is the same value we obtain for runs with an empty target chamber.

\section{DISCUSSIONS}

\section{A. Phase and density dependence}

From the present systematic studies we have obtained the following results.

\section{Gas phase}

DATS in low temperature gas at $10-30 \mathrm{~K}$ show the longest $T_{a v}$ of $4.03 \pm 0.02 \mu \mathrm{s}$. In this range there is no change of DATS at different densities. They are characterized by a downward bending in $\ln N(t)$ vs $t$ at later times as well as by the absence of a fast decaying component, whereas the previous DATS taken in room temperature gas and in liquid helium always showed fast decaying components and shorter $T_{a v}$ (around $3 \mu \mathrm{s}$ ). The absence of short-lived components at low temperatures indicates that there is no short-lived state of this lifetime range $(\sim 0.3 \mu \mathrm{s})$ that is populated directly; the states that are populated are either long lived (lifetime $\sim 1-2$ $\mu \mathrm{s}$ ) or Auger dominated (lifetime $\leq 10 \mathrm{~ns}$ ). Thus the fast decaying component as well as the shorter average lifetime in room temperature gas may be attributed either

TABLE I. Summary of target conditions, average lifetimes, and fitted decay rates for helium gas. The errors quoted for molar volume and density originate from uncertainties in measuring and stabilizing temperature and pressure of the gas samples. Two spectra of previous data which have been analyzed in the same way are shown for comparison.

\begin{tabular}{ccccccrc}
\hline \hline $\begin{array}{c}T \\
(\mathrm{~K})\end{array}$ & $\begin{array}{c}p \\
(\mathrm{bar})\end{array}$ & $\begin{array}{c}V_{\text {mol }} \\
\left(\mathrm{cm}^{3}\right)\end{array}$ & $\begin{array}{c}\rho \\
\left(10^{20} \mathrm{~cm}^{-3}\right)\end{array}$ & $\begin{array}{c}T_{a v}(150 \mathrm{~ns}) \\
(\mu \mathrm{s})\end{array}$ & $\begin{array}{c}T_{a v}(1 \mu \mathrm{s}) \\
(\mu \mathrm{s})\end{array}$ & $\begin{array}{c}\lambda_{1} \\
\left(\mu \mathrm{s}^{-1}\right)\end{array}$ & $\begin{array}{c}\lambda_{3} \\
\left(\mu \mathrm{s}^{-1}\right)\end{array}$ \\
\hline 10 & \multicolumn{7}{c}{ Low temperature ${ }^{4}$ He gas } \\
20 & 1 & $820 \pm 80$ & $7.53 \pm 0.75$ & $4.12 \pm 0.03$ & $3.97 \pm 0.03$ & $0.395 \pm 0.005$ \\
30 & 1 & $1640 \pm 170$ & $3.67 \pm 0.38$ & $4.22 \pm 0.03$ & $4.05 \pm 0.04$ & $0.401 \pm 0.005$ & $0.596 \pm 0.009$ \\
& 1 & $2460 \pm 250$ & $2.45 \pm 0.25$ & $4.16 \pm 0.02$ & $4.07 \pm 0.02$ & $0.376 \pm 0.003$ & $0.628 \pm 0.006$
\end{tabular}

Previous room temperature ${ }^{4} \mathrm{He}$ gas data

\begin{tabular}{lllllll}
\hline 300 & 3 & $7470 \pm 200$ & $0.81 \pm 0.02$ & $3.08 \pm 0.03$ & $3.18 \pm 0.04$ & $0.441 \pm 0.004$
\end{tabular}

Previous room temperature ${ }^{3} \mathrm{He}$ gas data

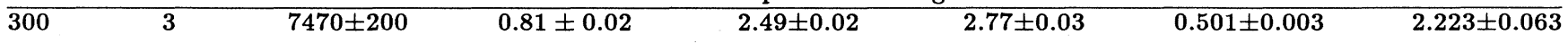


TABLE II. Summary of target conditions, average lifetimes, and fitted decay rates for liquid and solid helium. The errors of the molar volume are due to uncertainties in interpolating the experimental data.

\begin{tabular}{|c|c|c|c|c|c|c|c|c|}
\hline Remarks & $\begin{array}{c}T \\
(\mathrm{~K}) \\
\end{array}$ & $\begin{array}{c}p \\
\text { (bar) }\end{array}$ & $\begin{array}{l}V_{m o l} \\
\left(\mathrm{~cm}^{3}\right)\end{array}$ & $\begin{array}{c}\rho \\
\left(10^{22} \mathrm{~cm}^{-3}\right)\end{array}$ & $\begin{array}{c}T_{a v}(150 \mathrm{~ns}) \\
(\mu \mathrm{s})\end{array}$ & $\begin{array}{c}T_{a v}(1 \mu \mathrm{s}) \\
(\mu \mathrm{s})\end{array}$ & $\begin{array}{c}\lambda_{1} \\
\left(\mu \mathrm{s}^{-1}\right)\end{array}$ & $\begin{array}{c}\lambda_{3} \\
\left(\mu \mathrm{s}^{-1}\right)\end{array}$ \\
\hline \multicolumn{9}{|c|}{ liquid and solid ${ }^{4} \mathrm{He}$} \\
\hline & 3.50 & 1.0 & 32.0 & $1.882 \pm 0.012$ & $2.60 \pm 0.02$ & $3.01 \pm 0.04$ & $0.485 \pm 0.003$ & $3.88 \pm 0.11$ \\
\hline & 2.90 & 1.3 & 27.7 & $2.174 \pm 0.016$ & $2.50 \pm 0.06$ & $2.88 \pm 0.10$ & $0.523 \pm 0.010$ & $3.67 \pm 0.28$ \\
\hline \multirow[t]{4}{*}{ superfluid } & 1.46 & 1.0 & 27.2 & $2.214 \pm 0.016$ & $2.54 \pm 0.03$ & $2.90 \pm 0.04$ & $0.502 \pm 0.004$ & $3.80 \pm 0.15$ \\
\hline & 3.36 & 10.5 & 25.5 & $2.362 \pm 0.019$ & $2.24 \pm 0.07$ & $2.60 \pm 0.11$ & $0.575 \pm 0.013$ & $3.93 \pm 0.38$ \\
\hline & 3.60 & 20.0 & 23.8 & $2.531 \pm 0.021$ & $2.24 \pm 0.06$ & $2.63 \pm 0.10$ & $0.563 \pm 0.011$ & $3.82 \pm 0.27$ \\
\hline & 3.60 & 48.5 & 21.5 & $2.801 \pm 0.026$ & $2.25 \pm 0.06$ & $2.66 \pm 0.11$ & $0.571 \pm 0.011$ & $3.29 \pm 0.23$ \\
\hline \multirow[t]{2}{*}{ solid } & 1.50 & 50.5 & 20.8 & $2.896 \pm 0.028$ & $1.83 \pm 0.02$ & $2.14 \pm 0.03$ & $0.691 \pm 0.005$ & $3.57 \pm 0.09$ \\
\hline & 3.00 & 60.0 & 20.6 & $2.924 \pm 0.028$ & $2.05 \pm 0.08$ & $2.54 \pm 0.13$ & $0.631 \pm 0.016$ & $3.36 \pm 0.24$ \\
\hline \multicolumn{9}{|c|}{ liquid ${ }^{3} \mathrm{He}$} \\
\hline & 2.50 & 1.1 & 39.5 & $1.525 \pm 0.008$ & $2.65 \pm 0.05$ & $2.92 \pm 0.08$ & $0.483 \pm 0.008$ & $2.44 \pm 0.15$ \\
\hline & 2.35 & 1.3 & 38.0 & $1.585 \pm 0.008$ & $2.59 \pm 0.08$ & $2.81 \pm 0.12$ & $0.508 \pm 0.012$ & $2.77 \pm 0.37$ \\
\hline & 2.66 & 5.6 & 34.0 & $1.771 \pm 0.010$ & $2.56 \pm 0.06$ & $2.76 \pm 0.08$ & $0.509 \pm 0.009$ & $2.38 \pm 0.17$ \\
\hline & 2.70 & 8.9 & 31.8 & $1.894 \pm 0.012$ & $2.45 \pm 0.05$ & $2.67 \pm 0.07$ & $0.533 \pm 0.008$ & $2.67 \pm 0.18$ \\
\hline & 2.60 & 12.4 & 31.0 & $1.943 \pm 0.013$ & $2.39 \pm 0.05$ & $2.55 \pm 0.07$ & $0.551 \pm 0.009$ & $2.34 \pm 0.15$ \\
\hline & 2.54 & 22.0 & 27.5 & $2.190 \pm 0.016$ & $2.25 \pm 0.05$ & $2.41 \pm 0.07$ & $0.577 \pm 0.009$ & $2.57 \pm 0.17$ \\
\hline & 2.52 & 31.0 & 25.8 & $2.334 \pm 0.018$ & $2.10 \pm 0.05$ & $2.23 \pm 0.08$ & $0.653 \pm 0.011$ & $2.27 \pm 0.14$ \\
\hline
\end{tabular}

to a higher temperature effect or to uncontrolled small impurity levels in the gas; the fast decaying component observed in room temperature gas is thus essentially different in origin from that in liquid and solid phases, as shown below.

\section{Liquid phase}

We have obtained DATS for liquid ${ }^{3} \mathrm{He}$ and ${ }^{4} \mathrm{He}$ over a wide range of pressures and densities. The DATS in

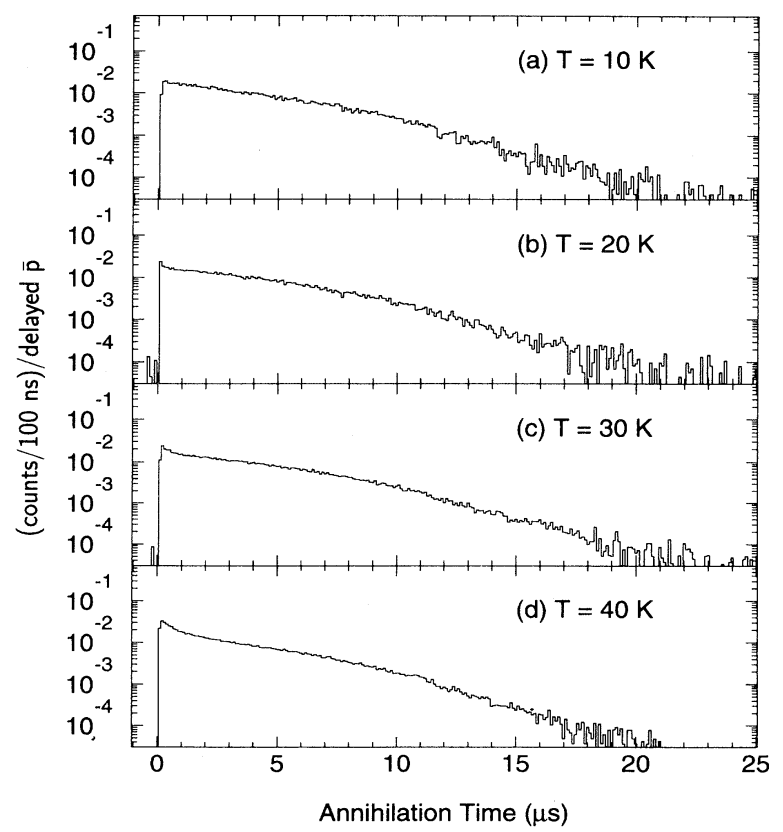

FIG. 6. DATS of ${ }^{4} \mathrm{He}$ gas between $10 \mathrm{~K}$ and $40 \mathrm{~K}$. liquid are generally composed of a fast decaying component (time constant $\sim 0.2 \mu \mathrm{s}$ ) and a long-lived component (time constant $\sim 2.5 \mu \mathrm{s}$ ). The $T_{a v}$ is substantially shorter than in low temperature gas and decreases slowly with increasing density, as shown in Fig. 9. All the liquid data can be represented by

$$
1 / T_{a v}=1 / T_{a v}^{(0)}+a \rho
$$

where $T_{a v}^{(0)}$ is the zero-density limit and $a$ is a density

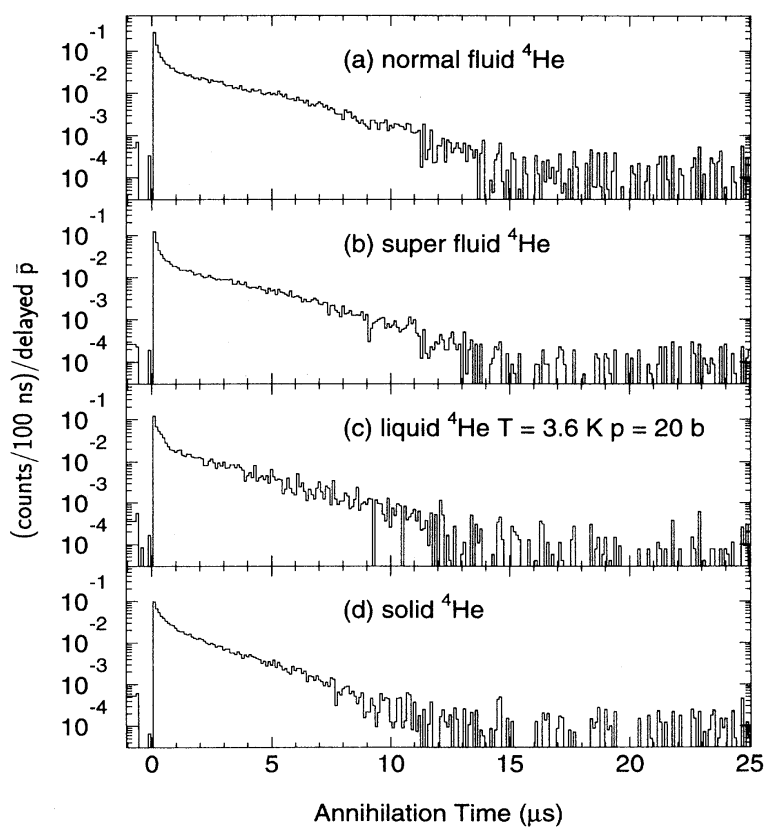

FIG. 7. Selected DATS of liquid and solid ${ }^{4} \mathrm{He}$. 


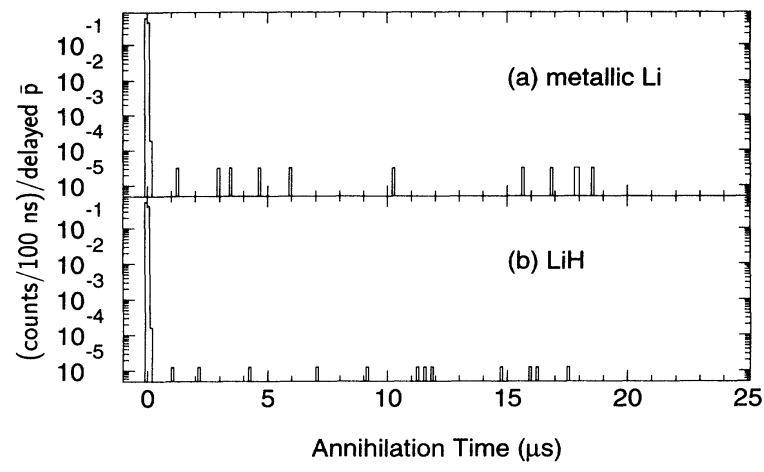

FIG. 8. DATS of multiplicity $\geq 3$ in solid lithium metal and lithium hydride.

coefficient. The best fitted values are $T_{a v}^{(0)}=4.45 \pm 0.27$ $\mu \mathrm{s}$ for ${ }^{4} \mathrm{He}$ and $T_{a v}^{(0)}=6.32 \pm 0.83 \mu \mathrm{s}$ for ${ }^{3} \mathrm{He}$ and $a=$ $(5.82 \pm 0.64) \times 10^{-24} \mathrm{~cm}^{3} / \mu \mathrm{s}$ for ${ }^{4} \mathrm{He}$ and $a=(1.19 \pm$ $0.11) \times 10^{-23} \mathrm{~cm}^{3} / \mu \mathrm{s}$ for ${ }^{3} \mathrm{He}$. The $T_{a v}(1 \mu \mathrm{s})$ in liquid ${ }^{4} \mathrm{He}$ is systematically longer than that in liquid ${ }^{3} \mathrm{He}$. The values at the same density, as shown in Table III, reveal an isotope dependence of the trapping time: the ratio of about 1.13 is close to the value observed in the gas phase [13].

The reduced $T_{a v}$ in liquid can be ascribed to the much higher density of the latter. The small linear density dependence of $T_{a v}(1 \mu \mathrm{s})$ in the liquid phase seems to be consistent with this view. On the contrary, the situation in the fast decaying component may be different, as will be discussed later. No difference was observed between the normal liquid phase at 1 bar and the superfluid phase.

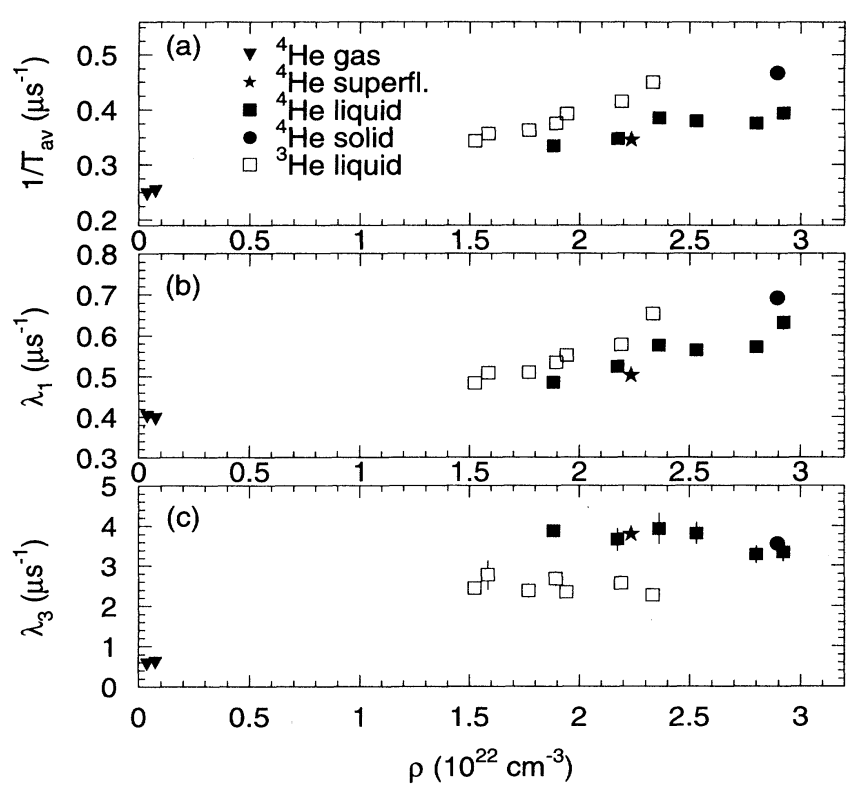

FIG. 9. Average decay rate $1 / T_{a v}(1 \mu \mathrm{s})$ and fitted decay rates $\lambda_{1}$ and $\lambda_{3}$ versus density $\rho$.

\section{Solid phase}

The DATS in solid helium shows a shape similar to that in liquid [see Fig. $7(\mathrm{~d})$ ], but a close look at $T_{a v}$ reveals a small but significant difference; the $T_{a v}$ in solid $\mathrm{He}(2.14 \pm 0.03 \mu \mathrm{s})$ is definitely shorter than that in liquid $\mathrm{He}$ at the same density $(2.54 \pm 0.04 \mu \mathrm{s})$. In Table IV we compare the $T_{a v}$ values of solid and liquid helium, six different points in liquid phase having been used to give a combined interpolated value at the solid helium density. This $20 \%$ effect looks striking in view of the small density dependence in liquid; the $T_{a v}$ in solid helium would correspond to a value in fictitious liquid of a very high density, 2-3 times the value at 1 bar (at enormously high pressure). The effect may require an explanation in terms of the phase change; the interaction of the $\bar{p} \mathrm{He}^{+}$atom with surrounding helium atoms is different in the solid and liquid phases.

\section{B. Chain decay model}

A careful look at the DATS suggests that $T_{a v}$ is not the best parameter to describe their shape in detail since they are not single exponentials; all except those for low-temperature gas exhibit a fast component of varying steepness followed by a downward-bending long-lived component. We therefore tried to find a more suitable function which can be fitted to the data, based on a simplified chain decay model. The following procedures can be justified in view of the selection rule (more appropriately, a propensity rule) of $\Delta n=\Delta l=-1$ for the radiative transitions $[7,9]$; namely, a state $(n, l)$ decays predominately to a state $(n-1, l-1)$ and so on. The entire DATS is then a superposition of mutually independent partial sequences to which a quantum number (radial node number or vibrational quantum number) $v=n-l-1$ can be assigned $(v=0$ for the circular sequence $l=n-1, v=1$ for the next circular sequence, etc.). Since we cannot discriminate the partial sequences by the shape of DATS alone, we try to approximate the ensemble of partial sequences by just one representative sequence. The parameters (populations and decay rates) to be deduced from such a procedure can be interpreted as in some sense an average over the ensemble of partial sequences.

The simplest assumption would be a sequence of two levels with decay rates $\lambda_{1}$ and $\lambda_{2}$; the first level feeds the second, from which the $\bar{p}$ proceeds to a Auger-dominated state followed by an Auger transition and subsequent nuclear capture and annihilation [see Fig. 10(a)]. The rate equations for the time-dependent populations, for levels 1 and $2, N_{1}(t)$ and $N_{2}(t)$, are

$$
\begin{aligned}
& \frac{d N_{1}}{d t}=-\lambda_{1} N_{1}, \\
& \frac{d N_{2}}{d t}=\lambda_{1} N_{1}-\lambda_{2} N_{2} .
\end{aligned}
$$

We assume that $\bar{p}$ annihilation takes place only from level 2 (i.e., we fit $\lambda_{2} N_{2}$ to our data). The solution for $N_{2}(t)$ is 
TABLE III. Ratios of average lifetimes and decay rates of ${ }^{3} \mathrm{He}$ to ${ }^{4} \mathrm{He}$ for liquid (this paper) and room temperature gas (previous data). In the case of a liquid the average of three measurements at almost identical density are given. The errors quoted for the trapping times $T_{\text {trap }}$ are systematic errors due to a dependence of the results on the fit range.

\begin{tabular}{lcccc}
\hline \hline & \multicolumn{3}{c}{ ratio ${ }^{3} \mathrm{He}$ to ${ }^{4} \mathrm{He}$} & $T_{\text {trap }}$ \\
\hline Condition & $T_{a v}(1 \mu s)$ & $\lambda_{1}$ & $\lambda_{3}$ & $1.13 \pm 0.02$ \\
gas & $1.15 \pm 0.03$ & $1.14 \pm 0.02$ & $1.76 \pm 0.09$ & $1.096 \pm 0.007$ \\
liquid & $1.15 \pm 0.03$ & $1.11 \pm 0.02$ & $0.66 \pm 0.05$ & 1.05 \\
\hline \hline
\end{tabular}

$$
\begin{aligned}
N_{2}(t)= & N_{1}(0) \frac{\lambda_{1}}{\lambda_{2}-\lambda_{1}} \exp \left(-\lambda_{1} t\right) \\
& +\left(N_{2}(0)-N_{1}(0) \frac{\lambda_{1}}{\lambda_{2}-\lambda_{1}}\right) \exp \left(-\lambda_{2} t\right)
\end{aligned}
$$

where $N_{i}(0)$ is the initial population of $\bar{p}$ for the level $i$.

The resulting spectrum is a sum of two exponentials. In fact, the two-level model fits the data of solid helium better than the three-level model to be described hereafter (lower right-hand side of Fig. 11). A fit to the data of such a simple function (dashed lines in Fig. 11), however, does not always give the best fit to the downward bending behavior, especially if the spectrum contains a fast component.

A model that can account for both the downward bend- ing observed in the time spectra and the fast component can easily be created by adding one level in the decay chain, as shown in Fig. 10(b). For this case the rate equations are $\left[N_{1}(t), N_{2}(t), N_{3}(t)\right.$ denote the populations and $\lambda_{1}, \lambda_{2}, \lambda_{3}$ the decay rates, respectively]

$$
\begin{aligned}
& \frac{d N_{1}}{d t}=-\lambda_{1} N_{1}, \\
& \frac{d N_{2}}{d t}=\lambda_{1} N_{1}-\lambda_{2} N_{2}, \\
& \frac{d N_{3}}{d t}=\lambda_{2} N_{2}-\lambda_{3} N_{3} .
\end{aligned}
$$

Again we assume that $\bar{p}$ annihilation takes place only from level 3 , i.e., we fit $\lambda_{3} N_{3}$ to our data. The solution for $N_{3}$ is

$$
\begin{aligned}
N_{3}(t)= & N_{1}(0) \frac{\lambda_{1} \lambda_{2}}{\left(\lambda_{2}-\lambda_{1}\right)\left(\lambda_{3}-\lambda_{1}\right)} \exp \left(-\lambda_{1} t\right)+\left(N_{2}(0)-N_{1}(0) \frac{\lambda_{1}}{\left(\lambda_{2}-\lambda_{1}\right)}\right) \frac{\lambda_{2}}{\left(\lambda_{3}-\lambda_{2}\right)} \exp \left(-\lambda_{2} t\right) \\
& +\left[N_{3}(0)-N_{1}(0) \frac{\lambda_{1} \lambda_{2}}{\left(\lambda_{2}-\lambda_{1}\right)\left(\lambda_{3}-\lambda_{1}\right)}-\left(N_{2}(0)-N_{1}(0) \frac{\lambda_{1}}{\lambda_{2}-\lambda_{1}}\right) \frac{\lambda_{2}}{\lambda_{3}-\lambda_{2}}\right] \exp \left(-\lambda_{3} t\right) .
\end{aligned}
$$

Since six free parameters are too many to obtain a consistent set of fit results in our case, we made the rather drastic assumption that the three levels are equally populated at $t=0$, i.e., $N_{1}(0)=N_{2}(0)=N_{3}(0)$. This assumption allows us to reduce the number of free parameters to 3 (plus overall normalization), i.e., the same as in the two-component exponential case.

We then found that we can successfully fit all the pure helium data using this function and that in most cases $\lambda_{1} \sim \lambda_{2}$. This observation motivated us to reduce even further the number of free parameters. By setting $\lambda_{1}=$ $\lambda_{2}, N_{3}(t)$ becomes

$$
\begin{aligned}
N_{3}(t)= & \left(-N_{1}(0) \frac{\lambda_{1}^{2}}{\left(\lambda_{3}-\lambda_{1}\right)^{2}}+N_{1}(0) \frac{\lambda_{1}^{2} t}{\lambda_{3}-\lambda_{1}}\right. \\
& \left.+N_{2}(0) \frac{\lambda_{1}}{\lambda_{3}-\lambda_{1}}\right) \exp \left(-\lambda_{1} t\right) \\
& +\left(N_{3}(0)+N_{1}(0) \frac{\lambda_{1}^{2}}{\left(\lambda_{3}-\lambda_{1}\right)^{2}}\right. \\
& \left.-N_{2}(0) \frac{\lambda_{1}}{\lambda_{3}-\lambda_{1}}\right) \exp \left(-\lambda_{3} t\right) .
\end{aligned}
$$

TABLE IV. Comparison of liquid and solid ${ }^{4} \mathrm{He}$.

\begin{tabular}{lccc}
\hline \hline Condition & $T_{a v}(1 \mu \mathrm{s})(\mu \mathrm{s})$ & $\lambda_{1}\left(\mu \mathrm{s}^{-1}\right)$ & $\lambda_{3}\left(\mu \mathrm{s}^{-1}\right)$ \\
\hline liquid & $2.54 \pm 0.04$ & $0.608 \pm 0.007$ & $3.37 \pm 0.13$ \\
solid & $2.14 \pm 0.03$ & $0.691 \pm 0.005$ & $3.57 \pm 0.09$ \\
\hline \hline
\end{tabular}

As stated above, we set $N_{1}(0)=N_{2}(0)=N_{3}(0)$ when we fit the data. Note that the second term [proportional to $\left.t \exp \left(-\lambda_{1} t\right)\right]$ is the growth-decay component.

Figure 11 shows the results of a logarithmic likelihood fit of this function to a wide variety of data, from low-

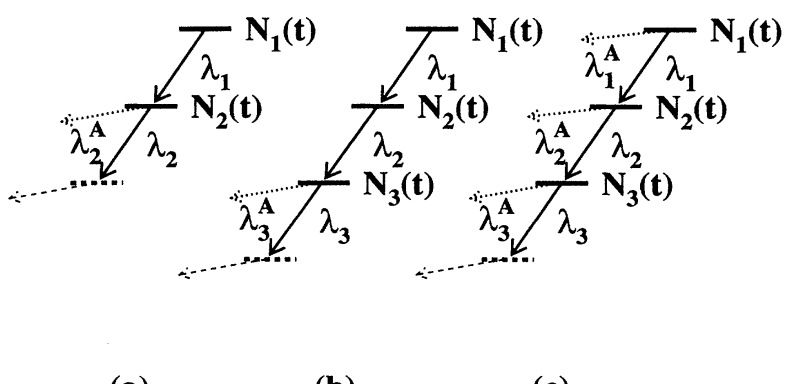

(a)

(b)

(c)

FIG. 10. Chain decay models for metastable state sequences: (a) with two metastable states, (b) with three metastable states, and (c) with three metastable states and additional destruction channels. Bold lines represent metastable levels and dashed lines or arrows Auger-dominated short-lived levels or transitions. $N_{i}(t)$ denote initial populations and $\lambda_{i}$ radiative decay rates. Dotted arrows represent destruction channels with rates $\lambda_{i}^{A}$. A change of the destruction rates $\lambda_{2}^{A}$ in (a) or $\lambda_{3}^{A}$ in (b) and (c) with helium density will result in a change of the fitted values for $\lambda_{2}$ and $\lambda_{3}$, even if the radiative lifetimes themselves do not change. 

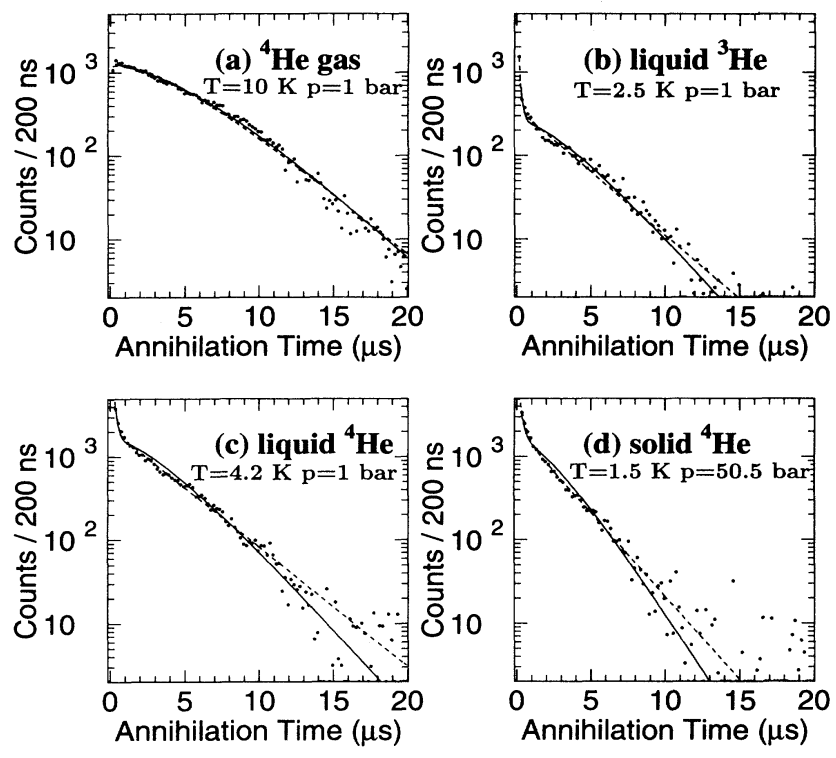

FIG. 11. Results of the two-level (dashed lines) and the three-level (solid lines) fit for a variety of conditions of $\mathrm{He}$ media.

temperature He gas to solid He. The quality of fitting is excellent for low-density data, but is somewhat poorer for solid and high pressure liquid. However, it is remarkable that such a simple function can fit all the data so well.

In Figs. 9(b) and 9(c), the two decay rates $\lambda_{1}$ and $\lambda_{3}$ are plotted against the density for all our data on pure helium. The $\lambda_{1}$ represents the slow lifetime component and shows an almost linear density dependence similar to the dependence of $T_{a v}^{-1}(1 \mu \mathrm{s})$ in the liquid phase [Fig. 9(a)]. Here again the $\lambda_{1}$ value in solid He is significantly larger than that in liquid $\mathrm{He}$ at the same density, as shown in Table IV. The values for liquid ${ }^{3} \mathrm{He}$ lie systematically above the ones of liquid ${ }^{4} \mathrm{He}$.

For $\lambda_{3}$, the fast lifetime component, the situation is different. The values for low temperature gas are very small, reflecting the absence of a visible fast component in those spectra [Fig. 9(c)]. For liquid helium, the values are fairly constant. The data of liquid ${ }^{3} \mathrm{He}$ lie far below those of liquid and solid ${ }^{4} \mathrm{He}$ indicating a significant difference in the fast component between the two isotopes.

\section{Chain decay model with destruction channel}

To improve the fitting and to include a mechanism that depends on collisions with other atoms, we added to the previous model a channel to immediate annihilation from levels 1 and 2 (e.g., to describe collisional destruction of the metastability), as shown in Fig. 10(c). The rate equations then become (setting for simplicity $\lambda^{A}=\lambda_{1}^{A}=$ $\lambda_{2}^{A}$ to be the decay rate to immediate annihilation)

$$
\begin{aligned}
& \frac{d N_{1}}{d t}=-\lambda_{1} N_{1}-\lambda^{A} N_{1} \\
& \frac{d N_{2}}{d t}=\lambda_{1} N_{1}-\lambda_{2} N_{2}-\lambda^{A} N_{2} \\
& \frac{d N_{3}}{d t}=\lambda_{2} N_{2}-\lambda_{3} N_{3}
\end{aligned}
$$

Now we have two more annihilation channels, so we fit $\lambda_{3} N_{3}+\lambda^{A} N_{1}+\lambda^{A} N_{2}$ to our data. With this model we obtained a somewhat improved fit to the data, but the results for the decay rates did not give a consistent picture for the density dependence of $\lambda^{A}$.

\section{Isotope effect}

In our first measurements of room temperature gas at LEAR $[13,16]$ we reported an isotope effect of the $\bar{p}$ trapping time: the time scale of the ${ }^{4} \mathrm{He}$ and ${ }^{3} \mathrm{He}$ gas spectra differ by $14 \pm 3 \%$. For liquid helium similar effects have been seen. As already mentioned in the previous sections, $T_{a v}, \lambda_{1}$, and $\lambda_{3}$ differ significantly for the two isotopes. Table III gives the average ratio for three points taken at almost the same density. The result for gaseous helium reported before [13] is shown for comparison. The ratio of the slow lifetime component, $T_{a v}(1 \mu s)$ and $\lambda_{1}$, are $1.16 \pm 0.02$ and $1.11 \pm 0.02$, respectively. Another procedure for comparing between ${ }^{3} \mathrm{He}$ and ${ }^{4} \mathrm{He}$ is to deduce the ratio of the trapping times $T_{\text {trap }}$ by enlarging the time scale of ${ }^{3} \mathrm{He}$. Here the time scale of the ${ }^{3} \mathrm{He}$ spectra has been stretched as described in [16] until it matches the ${ }^{4} \mathrm{He}$ spectra. Both procedures yield the same isotope ratio as shown in the last column of Table III.

\section{CONCLUSIONS}

In summary, we measured the delayed annihilation time spectra of antiprotons in various states of helium, from low temperature ${ }^{4} \mathrm{He}$ gas, high pressure liquid ${ }^{4} \mathrm{He}$ and ${ }^{3} \mathrm{He}$ to solid ${ }^{4} \mathrm{He}$ over a wide range of densities and obtained average lifetimes systematically. All spectra can be fairly well fitted with a simple three-level chain decay model. In spite of the many simplifying assumptions this model is able to reproduce the downward curved structure of the semilogarithmic spectra.

The low temperature gas shows the longest lifetime observed so far. The average lifetime as well as the slow component $\lambda_{1}$ of the fit model in gas and liquid exhibits a significant density dependence. This dependence, however, is very weak compared to the very large density difference between $10 \mathrm{~K}$ gas and liquid. The $\bar{p} \mathrm{He}^{+}$system seems to be quite insensitive to the presence of surrounding helium atoms, in sharp contrast to the strong effect observed previously of small quantities of hydrogen and other impurities.

The average decay rate as well as $\lambda_{1}$ in solid ${ }^{4} \mathrm{He}$ was, however, found to be significantly larger (by $~ 20 \%$ ) than it was in liquid ${ }^{4} \mathrm{He}$ of the same density. This observation raises an interesting question about the effect of the local environment on our $\bar{p} \mathrm{He}^{+}$atom. 
There is a marked difference in the fast component between low temperature gas and liquid or solid ${ }^{4} \mathrm{He}$. The DATS in gas phase show no fast component and the deduced $\lambda_{3}$ value for gas is very small compared with those in liquid; the data points show too big a jump to be connected smoothly between gas and liquid. The $\lambda_{3}$ values for ${ }^{3} \mathrm{He}$ are systematically smaller than those for ${ }^{4} \mathrm{He}$. Further studies of all these intriguing results are in progress.

The gross isotope effect, found previously in helium gas [13], has now been confirmed for liquid helium: the average lifetime as well as the $\lambda_{1}$ value of ${ }^{4} \mathrm{He}$ is about $10 \%$ larger than that of ${ }^{3} \mathrm{He}$ at the same density. This value reflects the difference of the radiative lifetimes of single levels caused by the difference in the reduced mass of the two isotopes [7].

A more sophisticated model including a decay channel to immediate annihilation (due to collisions, e.g.) was tried, but did not produce a better physical description of the data. Finally, we measured the delayed annihilation time spectra in solid lithium (Li-metal, $\mathrm{LiH}$, and $\mathrm{LiF}$ ), which give no evidence for a trapping effect for antiprotons in lithium (upper limit $\sim 0.015 \%$ ).

\section{ACKNOWLEDGMENTS}

We would like to thank the CERN-LEAR staff for their efforts in providing us with the stable high-quality lowmomentum antiproton beam. We also thank Dr. K. Ohtsuki for theoretical discussions. The present work is supported by the Grant-in-Aid for Specially Promoted Scientific Research and International Scientific Research of the Japanese Ministry of Education, Science and Culture and the International Joint Research Project of the Japan Society for the Promotion of Science (JSPS). Two of the authors (S.N.N. and E.W.) acknowledge financial support from JSPS.
[1] M. Iwasaki, S.N. Nakamura, K. Shigaki, Y. Shimizu, H. Tamura, T. Ishikawa, R.S. Hayano, E. Takada, E. Widmann, H. Outa, M. Aoki, P. Kitching, and T. Yamazaki, Phys. Rev. Lett. 67, 1246 (1991).

[2] T. Yamazaki, M. Aoki, M. Iwasaki, R.S. Hayano, T. Ishikawa, H. Outa, E. Takada, H. Tamura, and A. Sakaguchi, Phys. Rev. Lett. 63, 1590 (1989).

[3] S.N. Nakamura, M. Iwasaki, H. Outa, R.S. Hayano, Y. Watanabe, T. Nagae, T. Yamazaki, H. Tada, T. Numao, Y. Kuno, and R. Kadono, Phys. Rev. A 45, 6202 (1992).

[4] G.T. Condo, Phys. Lett. 9, 65 (1964).

[5] J.E. Russell, Phys. Rev. Lett. 23, 63 (1969); Phys. Rev. 188, 187 (1969); Phys. Rev. A 1, 721 (1970); 1, 735 (1970); 1, 742 (1970); J. Math. Phys. 12, 1906 (1971); Phys. Rev. A 6, 2488 (1972).

[6] R.S. Hayano, M. Iwasaki, and T. Yamazaki, in Perspectives of Meson Science, edited by T. Yamazaki, K. Nakai, and K. Nagamine (North-Holland, Amsterdam, 1992), p. 417.

[7] T. Yamazaki and K. Ohtsuki, Phys. Rev. A 45, 7782 (1992).

[8] R. Ahlrichs, O. Dumbrajs, H. Pilkuhn, and H.G. Schlaile, Z. Phys. A 306, 297 (1982).

[9] I. Shimamura, Phys. Rev. A 46, 3376 (1992).

[10] P.T. Greenland and R. Thürwächter, Hyperfine Interact. 76, 355 (1993).

[11] K. Ohtsuki (private communication).
[12] N. Morita, K. Ohtsuki, and T. Yamazaki, Nucl. Instrum. Methods Phys. Res., Sect. A 330, 439 (1993).

[13] T. Yamazaki, E. Widmann, R.S. Hayano, M. Iwasaki, S.N. Nakamura, K. Shigaki, F.J. Hartmann, H. Daniel, T. von Egidy, P. Hofmann, Y.-S, Kim, and J. Eades, Nature 361, 238 (1993).

[14] N. Morita, M. Kamakura, T. Yamazaki, E. Widmann, H. Masuda, I. Sugai, R.S. Hayano, F.E. Maas, H.A. Torii, F.J. Hartmann, H. Daniel, T. von Egidy, B. Ketzer, W. Müller, W. Schmid, D. Horvath, and J. Eades, Phys. Rev. Lett. 72, 1180 (1994).

[15] R.S. Hayano, F.E. Maas, H.A. Torii, N. Morita, M. Kamakura, T. Yamazaki, H. Masuda, I. Sugai, F.J. Hartmann, H. Daniel, T. von Egidy, B. Ketzer, W. Müller, W. Schmid, D. Horvath, J. Eades, and E. Widmann, Phys. Rev. Lett. 73, 1485 (1994); 73, 3181 (1994) (E).

[16] S.N. Nakamura, R.S. Hayano, M. Iwasaki, K. Shigaki, E. Widmann, T. Yamazaki, H. Daniel, T. von Egidy, F.J. Hartmann, P. Hofmann, Y.-S. Kim, and J. Eades, Phys. Rev. A 49, 4457 (1994).

[17] W.H. Keesom, Helium (Elsevier, Amsterdam, 1942).

[18] C. Domb and J.S. Dugdale, Prog. Low Temp. Phys. 2, 338 (1957); E.R. Grilly and R.L. Mills, Ann. Phys. (N.Y.) 18, 250 (1962).

[19] American Institute of Physics Handbook, edited by Dwight E. Gray (McGraw-Hill, New York, 1972), pp. 4$298 \mathrm{ff}$. 


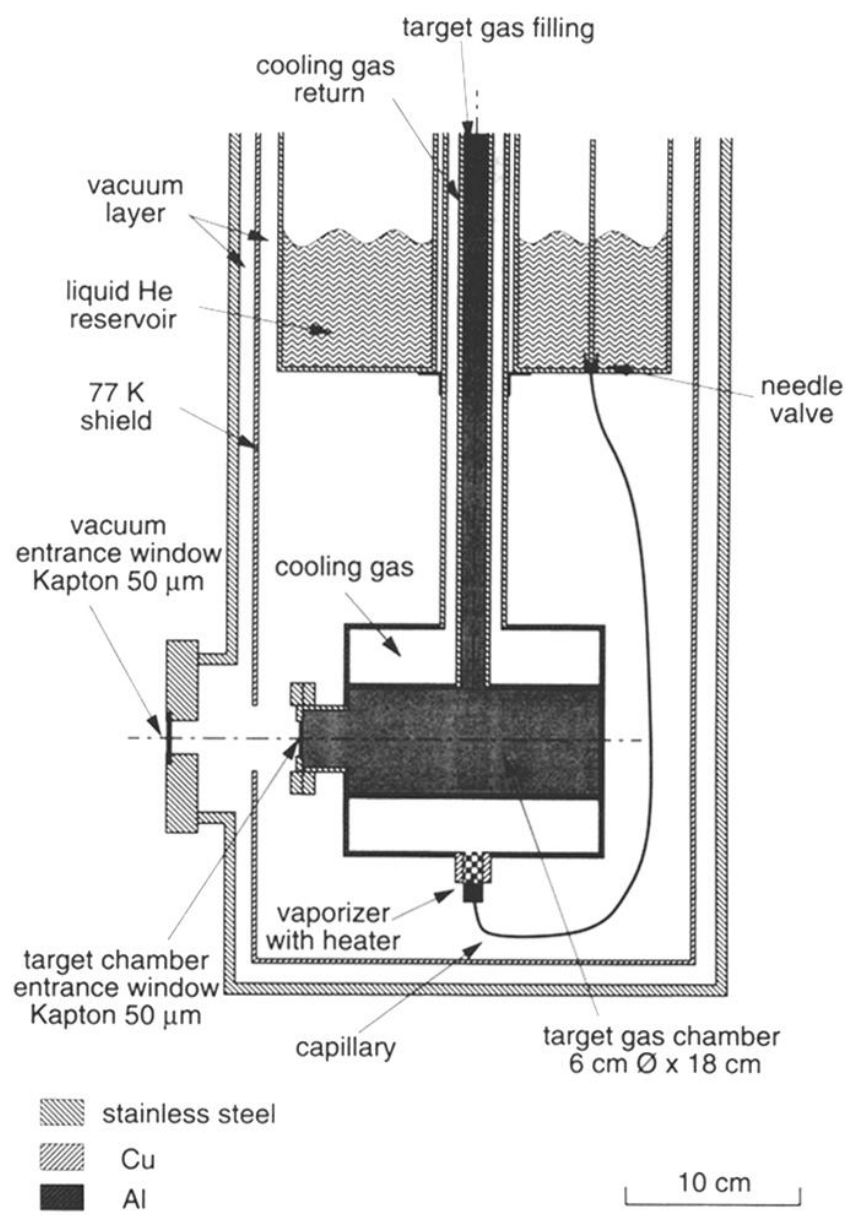

FIG. 2. Cryostat for low temperature gas measurements (side view). In the upper part the liquid helium reservoir with a needle valve to control the flow of liquid helium through the capillary to the vaporizer is shown. In the lower part the target gas chamber and the surrounding cooling gas chamber are seen. The antiproton beam came from the left-hand side. 